Supplement of Earth Syst. Sci. Data, 12, 1775-1787, 2020

乡 Earth System

https://doi.org/10.5194/essd-12-1775-2020-supplement

(c) Author(s) 2020. This work is distributed under

the Creative Commons Attribution 4.0 License.

(c) (1)

Supplement of

\title{
Reanalysis of vertical mixing in mesocosm experiments: PeECE III and KOSMOS 2013
}

\section{Sabine Mathesius et al.}

Correspondence to: Sabine Mathesius (sabine_mathesius@sfu.ca)

The copyright of individual parts of the supplement might differ from the CC BY 4.0 License. 

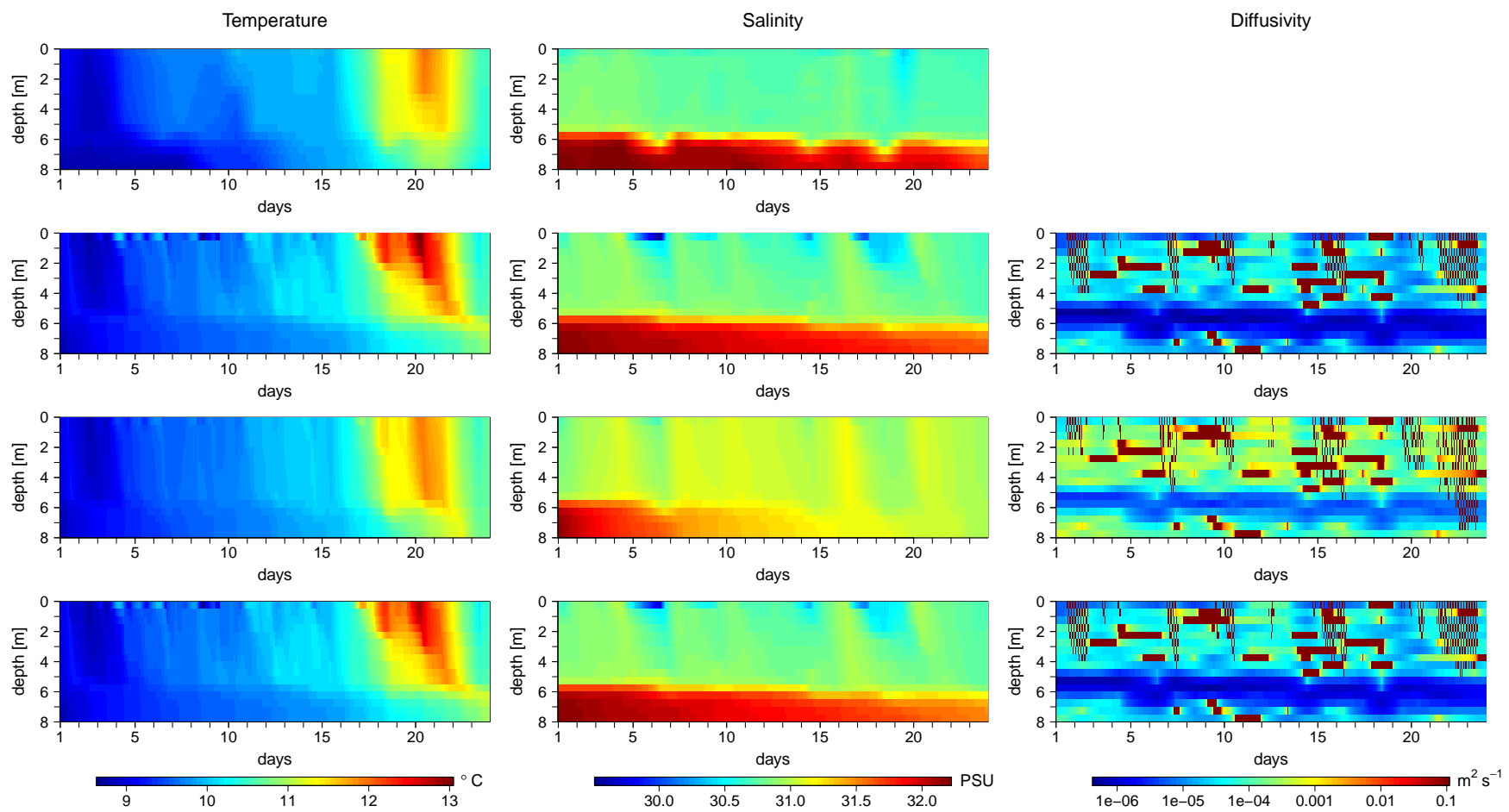

Figure S 1. Temporal evolution of temperature, salinity, and diffusivity profiles in mesocosm \#1 of PeECE III. The first row shows observed temperature and salinity; the following rows show simulated temperature, salinity, and diffusivity for a) the salinity optimisation (second row), b) the temperature optimisation (third row), and c) the optimisation that depends on both temperature and salinity (fourth row).
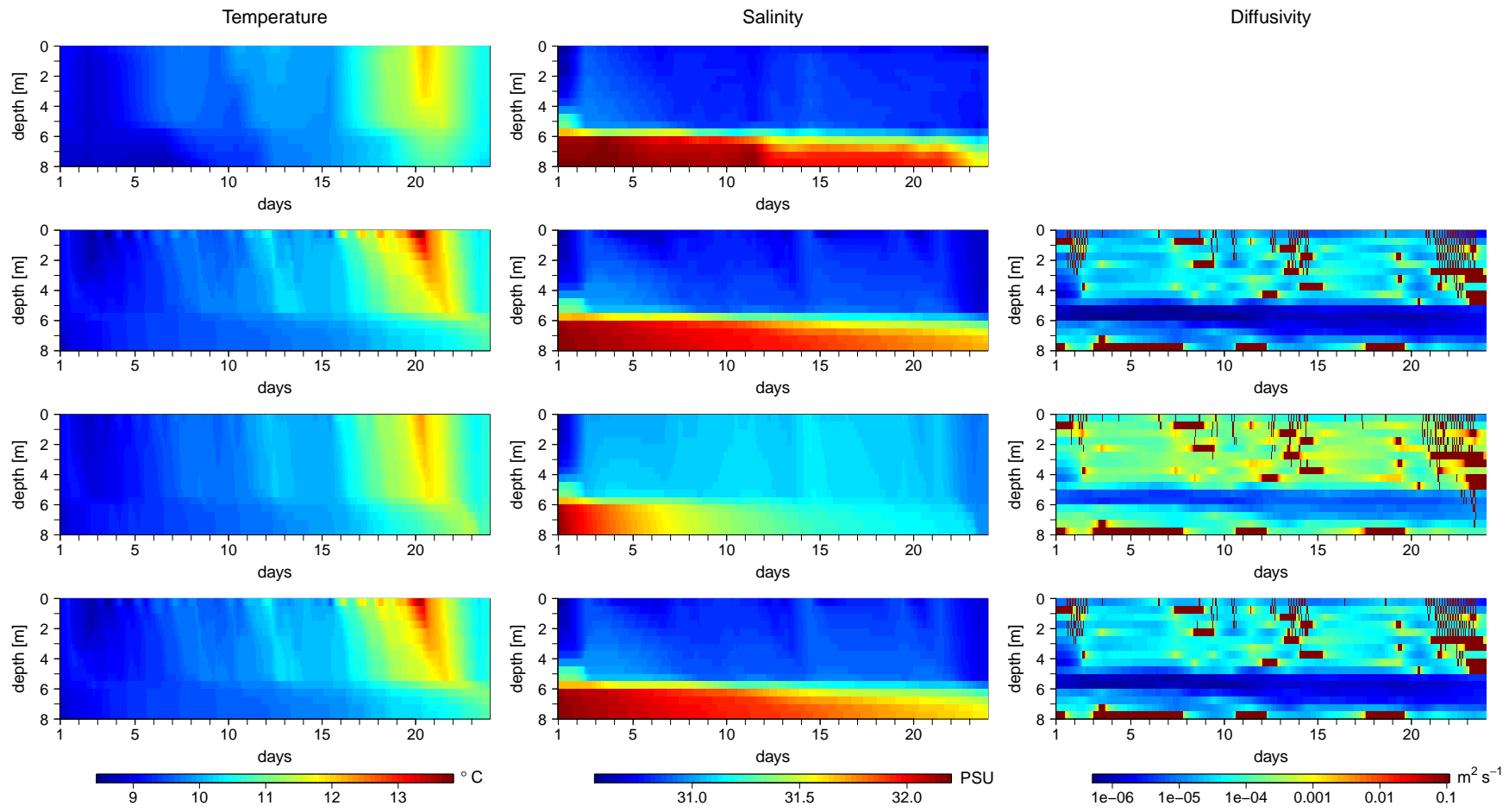

Figure S 2. Temporal evolution of temperature, salinity, and diffusivity profiles in mesocosm \#2 of PeECE III. The first row shows observed temperature and salinity; the following rows show simulated temperature, salinity, and diffusivity for a) the salinity optimisation (second row), b) the temperature optimisation (third row), and c) the optimisation that depends on both temperature and salinity (fourth row). 

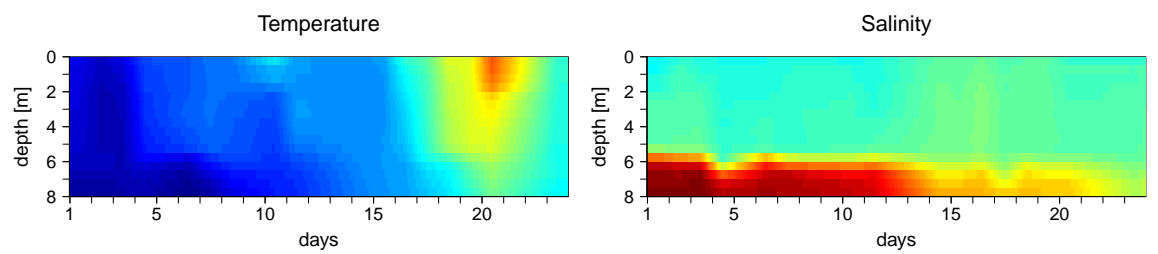

Diffusivity
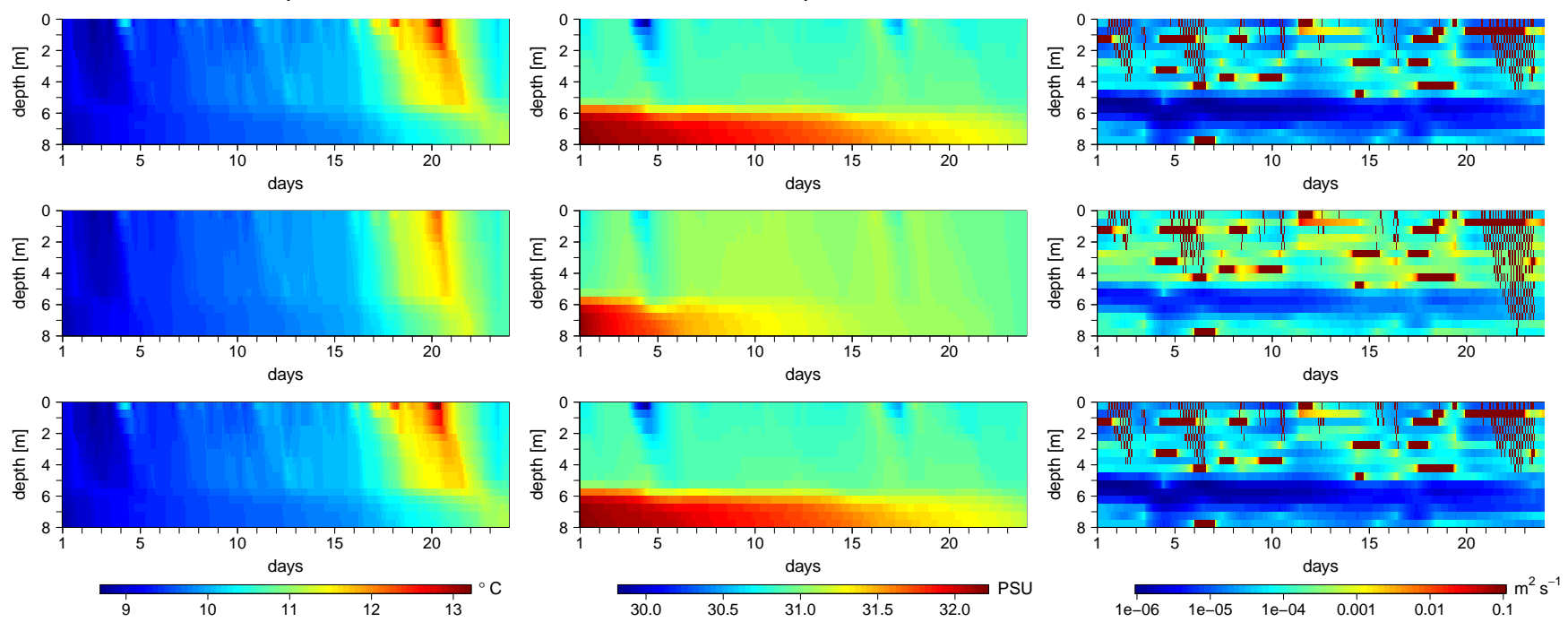

Figure S 3. Temporal evolution of temperature, salinity, and diffusivity profiles in mesocosm \#3 of PeECE III. The first row shows observed temperature and salinity; the following rows show simulated temperature, salinity, and diffusivity for a) the salinity optimisation (second row), b) the temperature optimisation (third row), and c) the optimisation that depends on both temperature and salinity (fourth row).
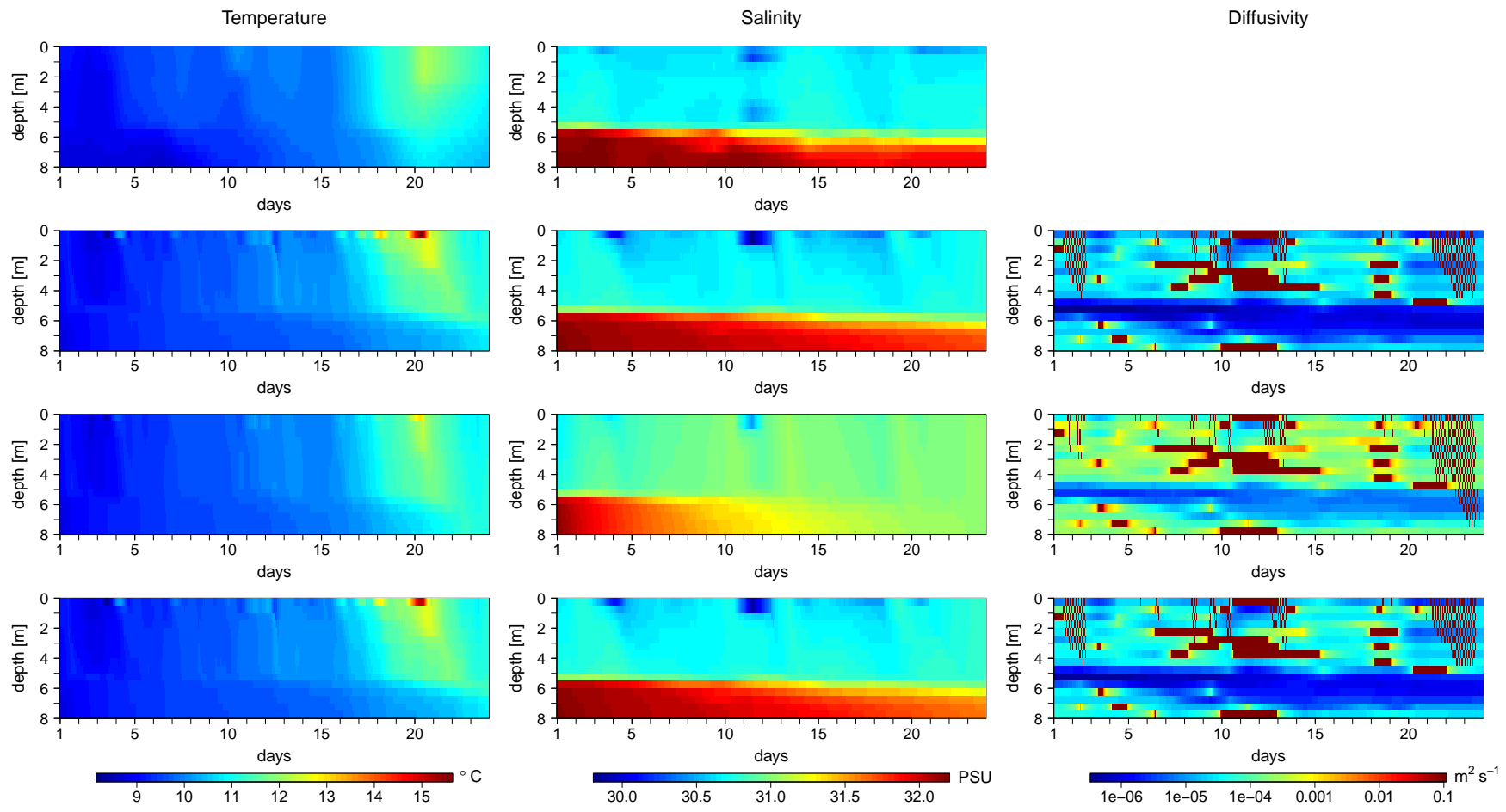

Figure S 4. Temporal evolution of temperature, salinity, and diffusivity profiles in mesocosm \#4 of PeECE III. The first row shows observed temperature and salinity; the following rows show simulated temperature, salinity, and diffusivity for a) the salinity optimisation (second row), b) the temperature optimisation (third row), and c) the optimisation that depends on both temperature and salinity (fourth row). 

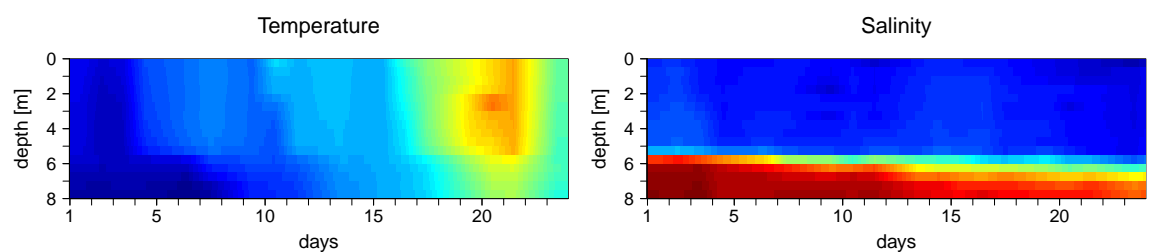

Diffusivity
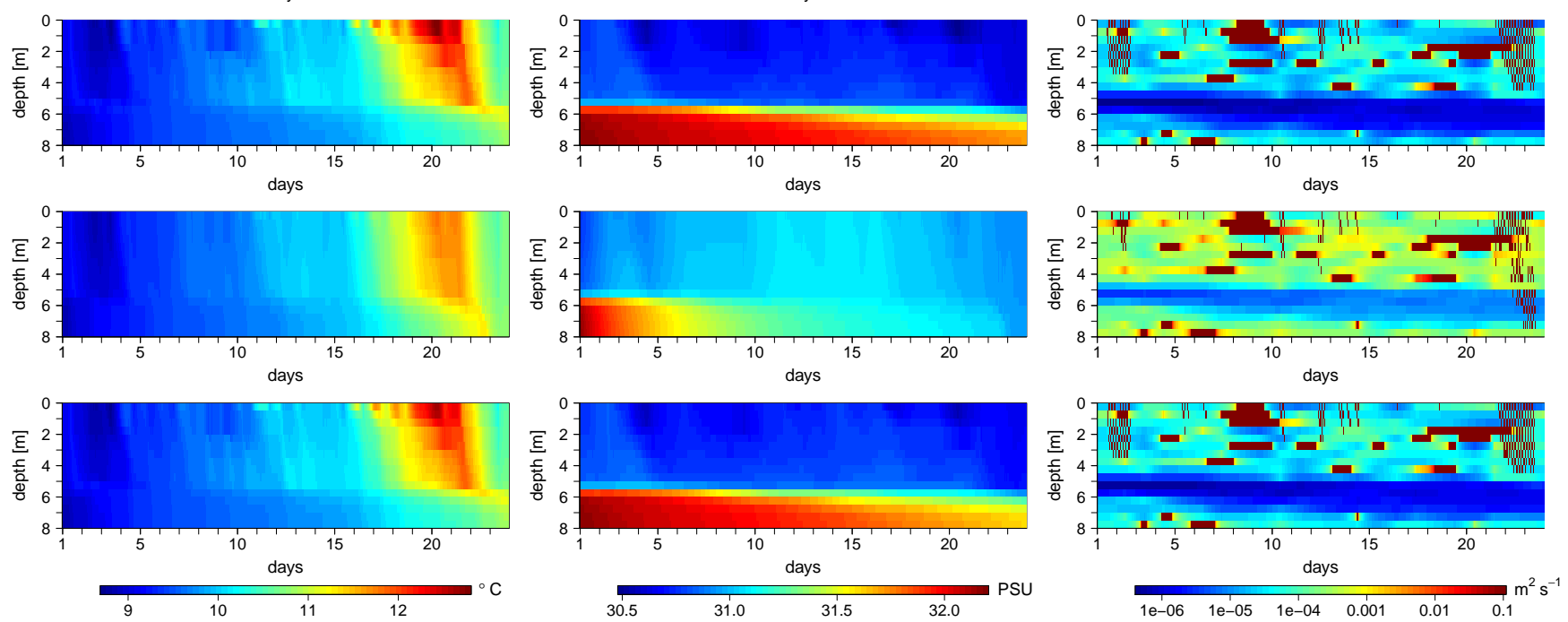

Figure S 5. Temporal evolution of temperature, salinity, and diffusivity profiles in mesocosm \#5 of PeECE III. The first row shows observed temperature and salinity; the following rows show simulated temperature, salinity, and diffusivity for a) the salinity optimisation (second row), b) the temperature optimisation (third row), and c) the optimisation that depends on both temperature and salinity (fourth row).
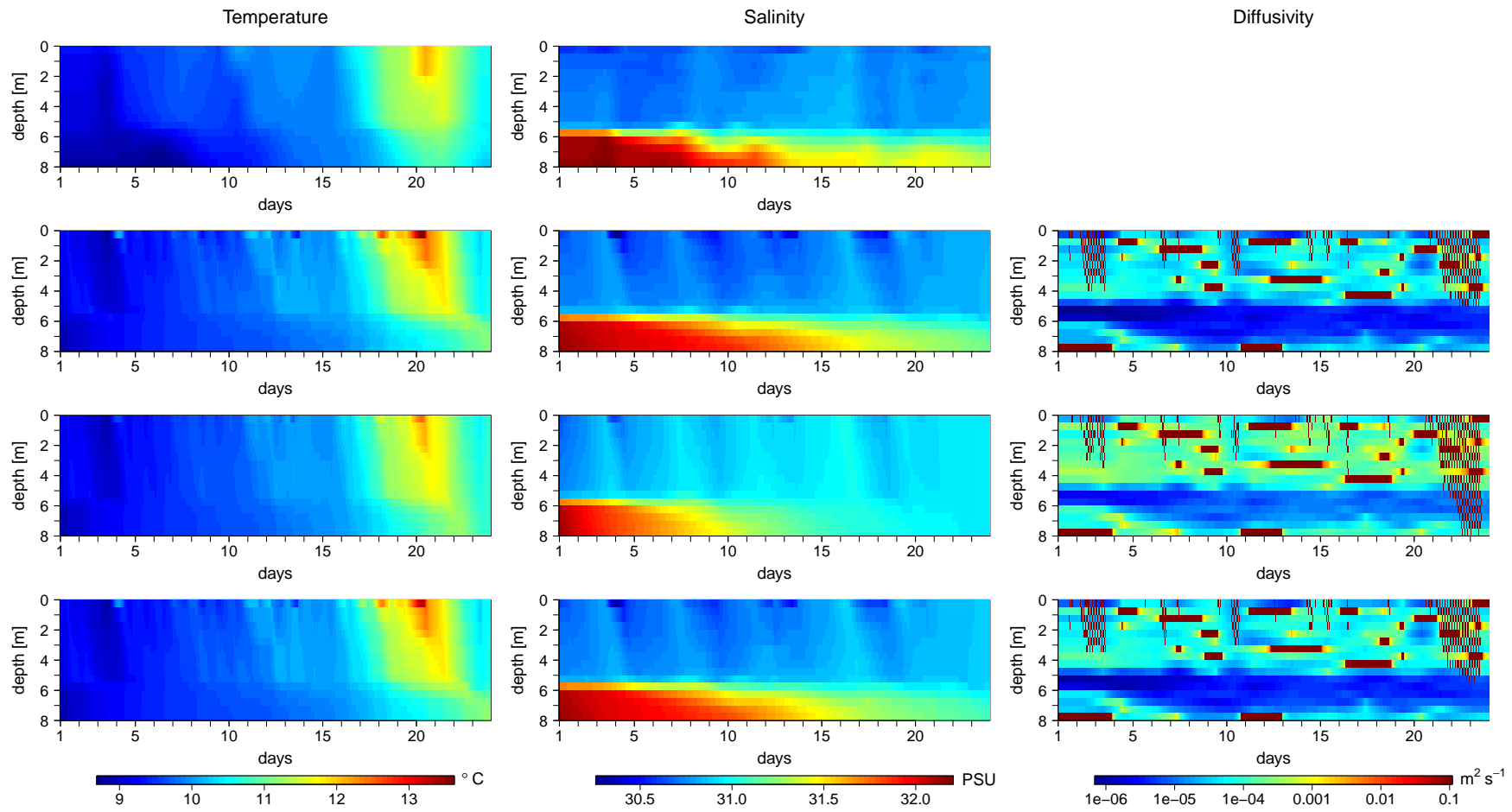

Figure S 6. Temporal evolution of temperature, salinity, and diffusivity profiles in mesocosm \#6 of PeECE III. The first row shows observed temperature and salinity; the following rows show simulated temperature, salinity, and diffusivity for a) the salinity optimisation (second row), b) the temperature optimisation (third row), and c) the optimisation that depends on both temperature and salinity (fourth row). 

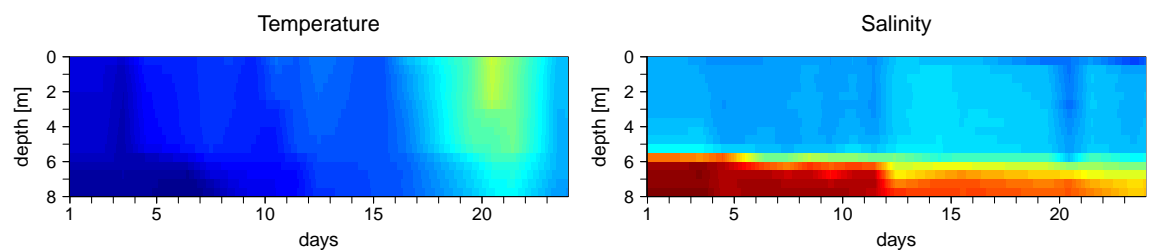

Diffusivity
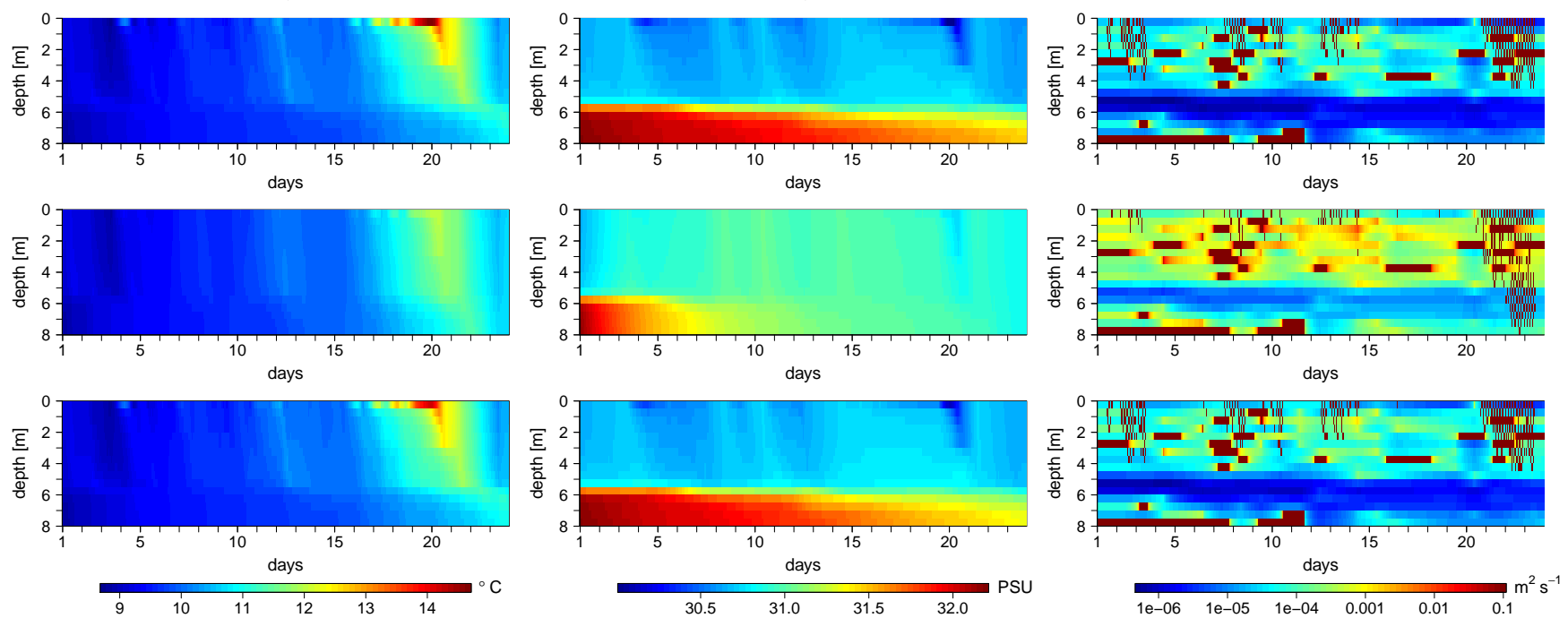

Figure S 7. Temporal evolution of temperature, salinity, and diffusivity profiles in mesocosm \#7 of PeECE III. The first row shows observed temperature and salinity; the following rows show simulated temperature, salinity, and diffusivity for a) the salinity optimisation (second row), b) the temperature optimisation (third row), and c) the optimisation that depends on both temperature and salinity (fourth row).
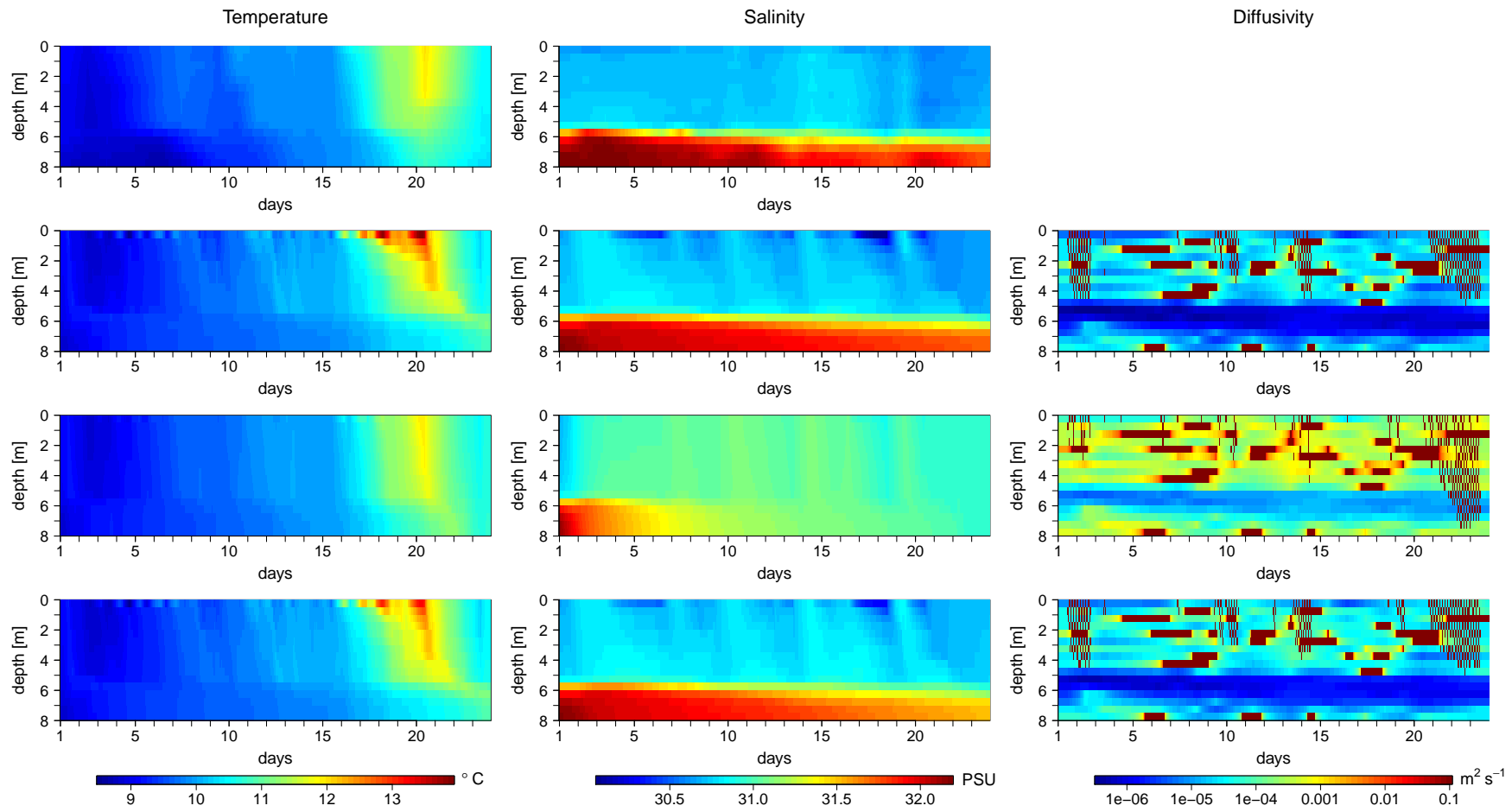

Figure S 8. Temporal evolution of temperature, salinity, and diffusivity profiles in mesocosm \#8 of PeECE III. The first row shows observed temperature and salinity; the following rows show simulated temperature, salinity, and diffusivity for a) the salinity optimisation (second row), b) the temperature optimisation (third row), and c) the optimisation that depends on both temperature and salinity (fourth row). 

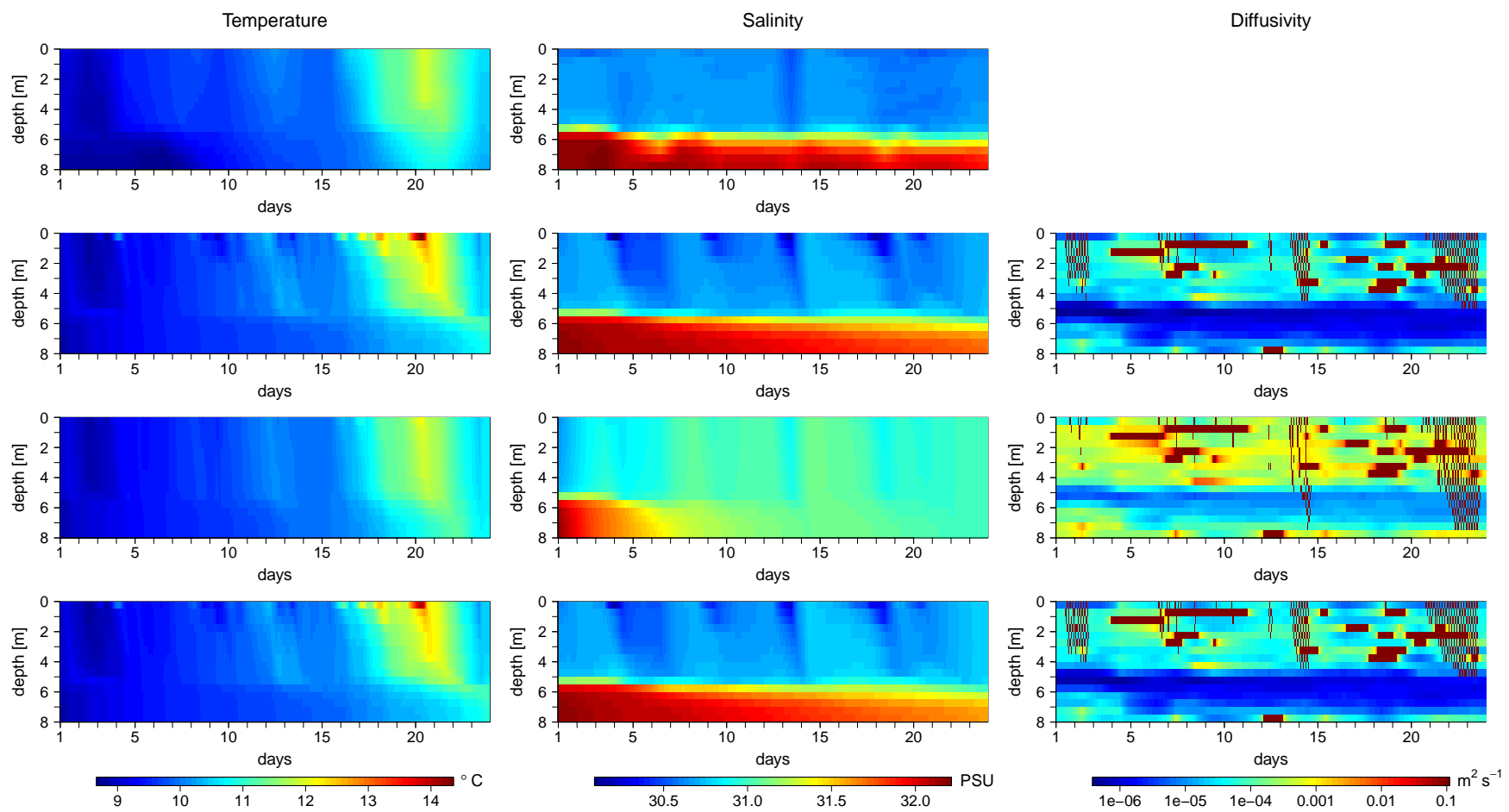

Figure S 9. Temporal evolution of temperature, salinity, and diffusivity profiles in mesocosm \#9 of PeECE III. The first row shows observed temperature and salinity; the following rows show simulated temperature, salinity, and diffusivity for a) the salinity optimisation (second row), b) the temperature optimisation (third row), and c) the optimisation that depends on both temperature and salinity (fourth row).
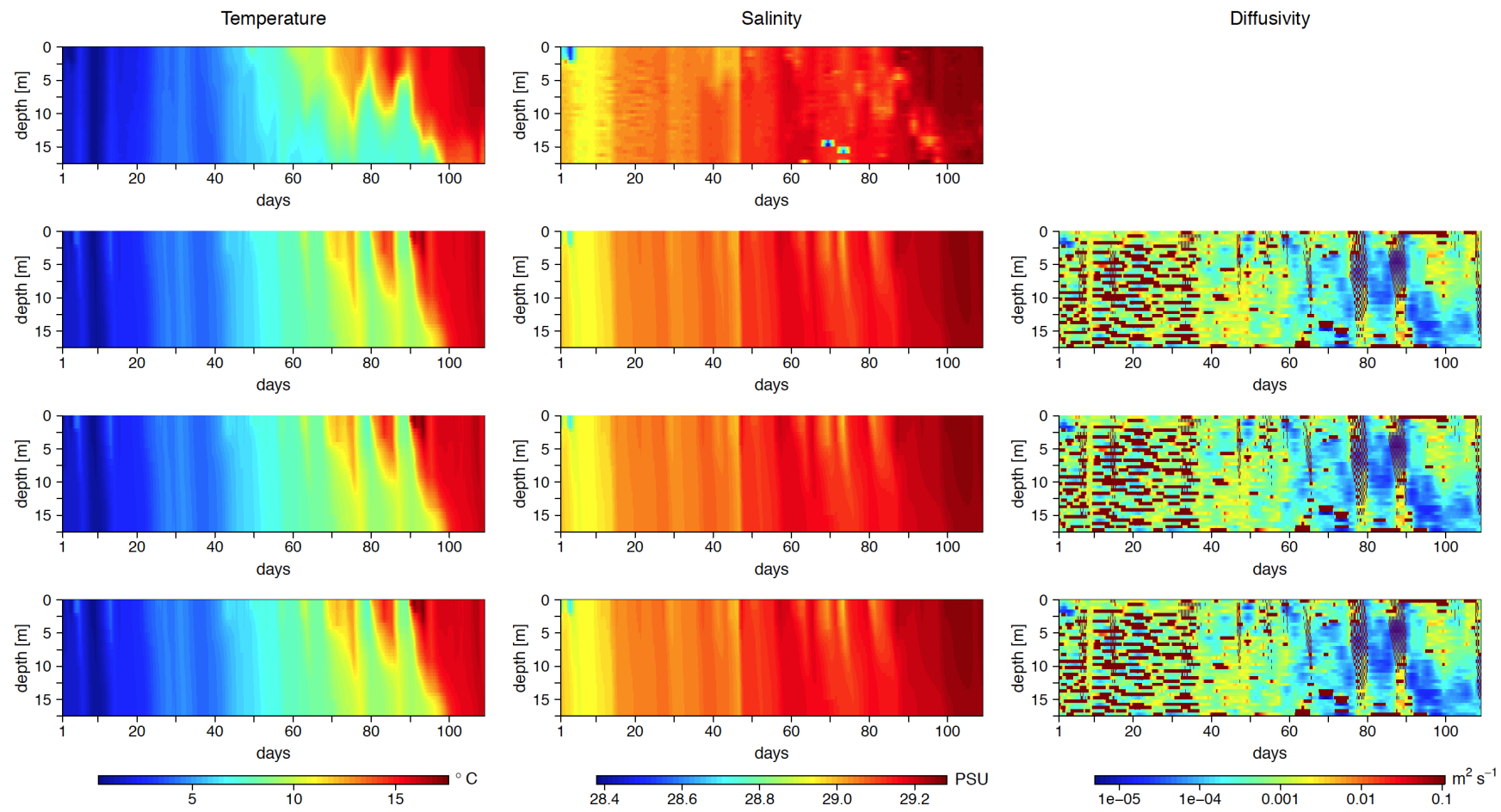

Figure S 10. Temporal evolution of temperature, salinity, and diffusivity profiles in mesocosm \#1 of KOSMOS 2013. The first row shows observed temperature and salinity; the following rows show simulated temperature, salinity, and diffusivity for a) the salinity optimisation (second row), b) the temperature optimisation (third row), and c) the optimisation that depends on both temperature and salinity (fourth row). 

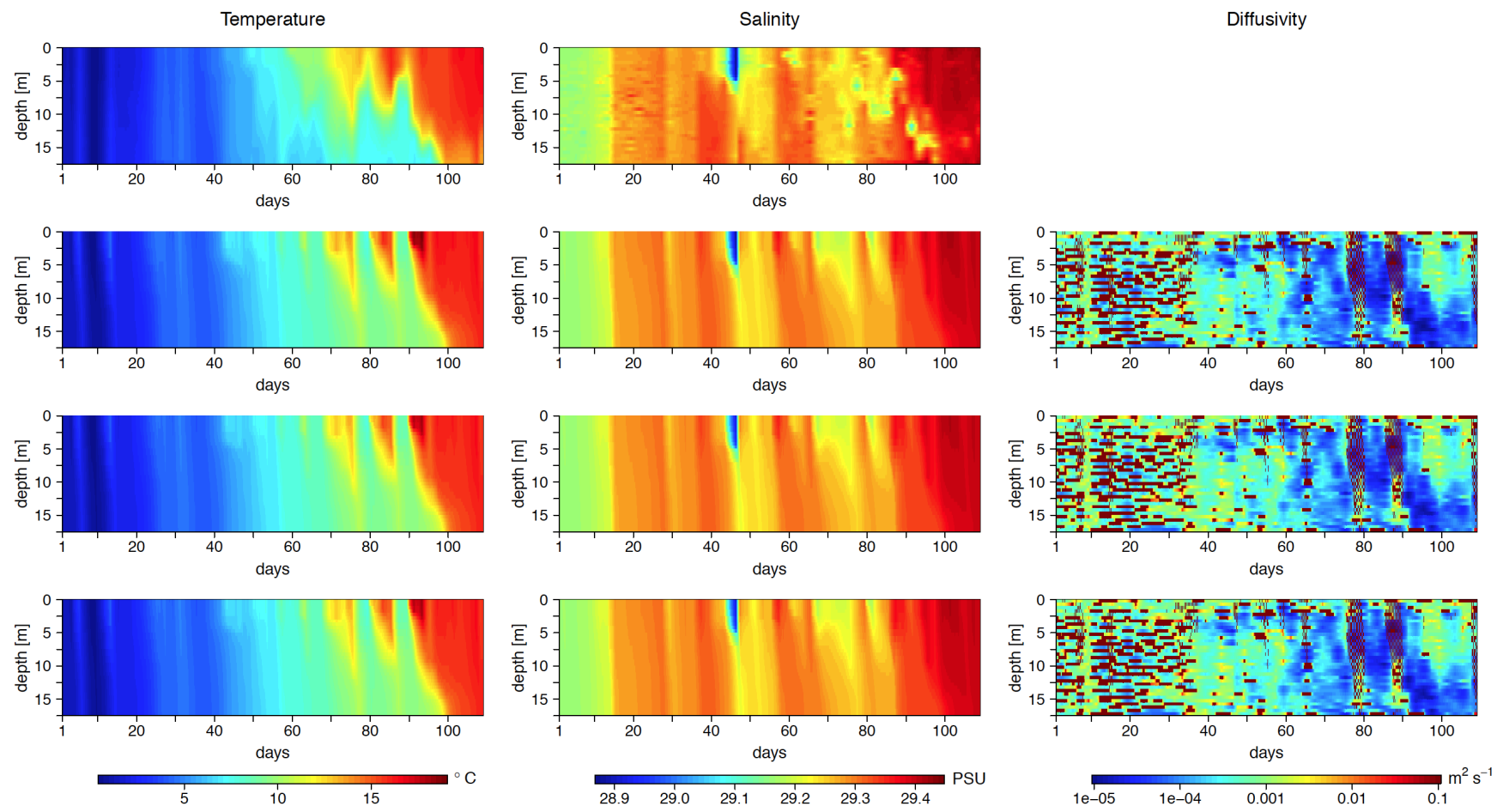

Figure S 11. Temporal evolution of temperature, salinity, and diffusivity profiles in mesocosm \#2 of KOSMOS 2013. The first row shows observed temperature and salinity; the following rows show simulated temperature, salinity, and diffusivity for a) the salinity optimisation (second row), b) the temperature optimisation (third row), and c) the optimisation that depends on both temperature and salinity (fourth row).
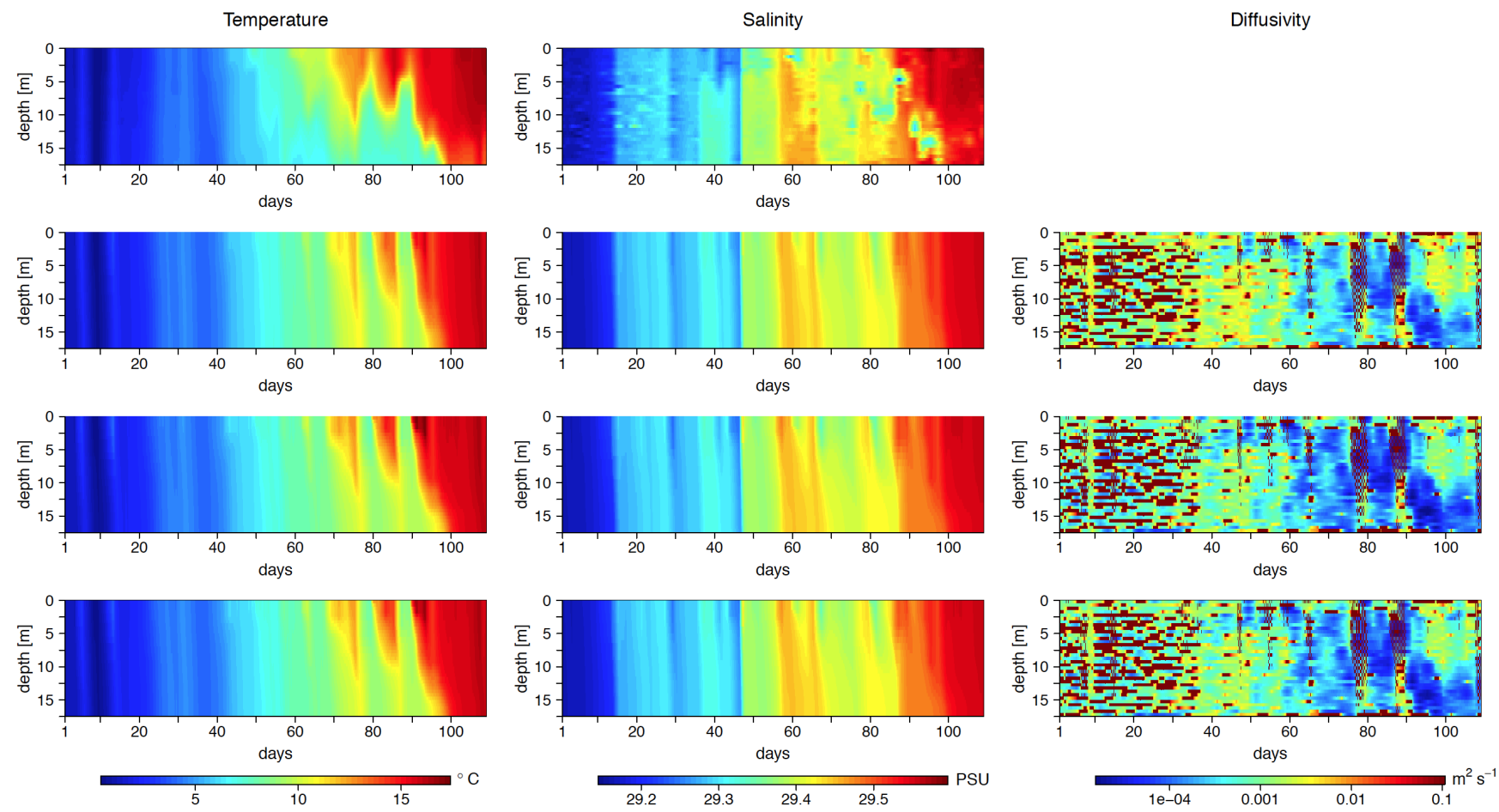

Figure S 12. Temporal evolution of temperature, salinity, and diffusivity profiles in mesocosm \#3 of KOSMOS 2013. The first row shows observed temperature and salinity; the following rows show simulated temperature, salinity, and diffusivity for a) the salinity optimisation (second row), b) the temperature optimisation (third row), and c) the optimisation that depends on both temperature and salinity (fourth row). 

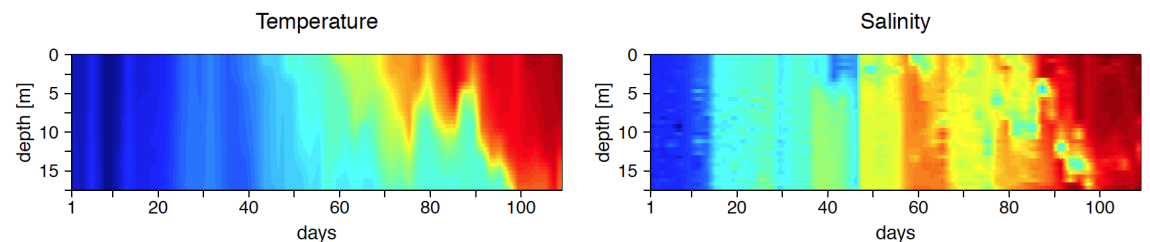

Diffusivity
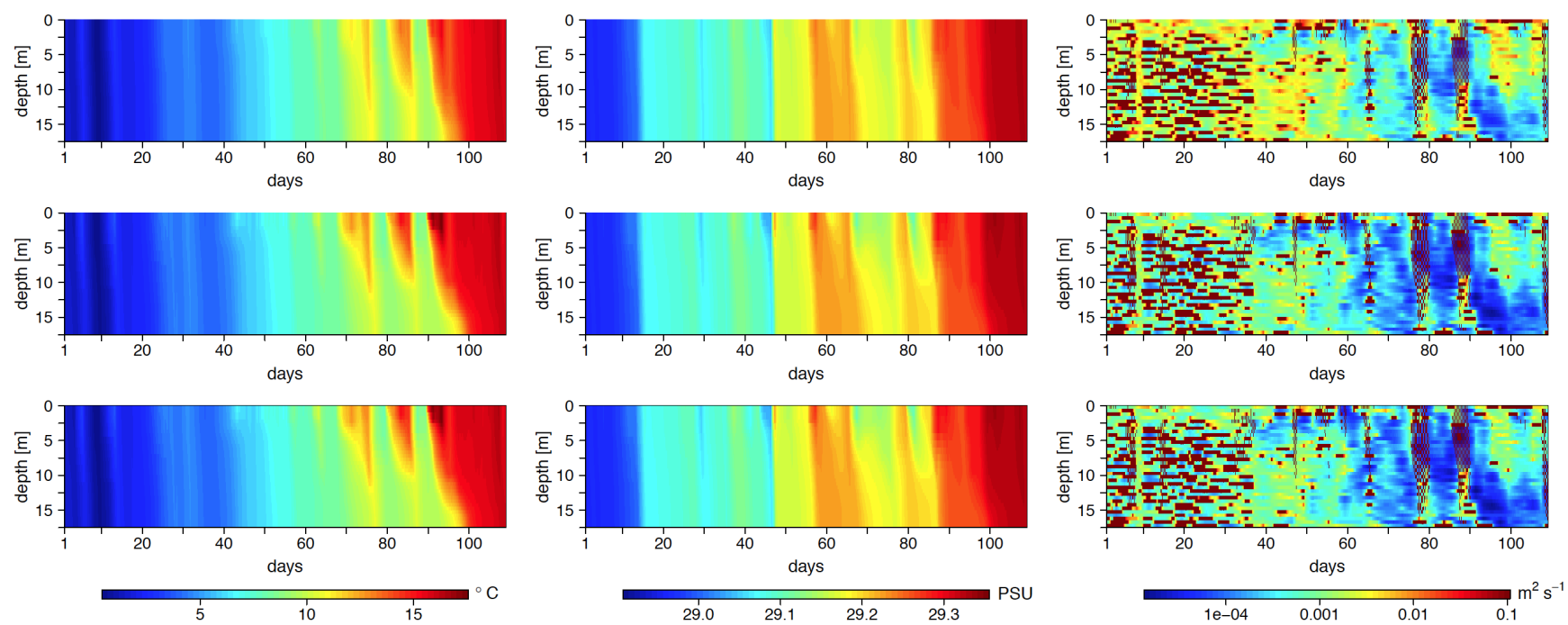

Figure S 13. Temporal evolution of temperature, salinity, and diffusivity profiles in mesocosm \#4 of KOSMOS 2013. The first row shows observed temperature and salinity; the following rows show simulated temperature, salinity, and diffusivity for a) the salinity optimisation (second row), b) the temperature optimisation (third row), and c) the optimisation that depends on both temperature and salinity (fourth row).
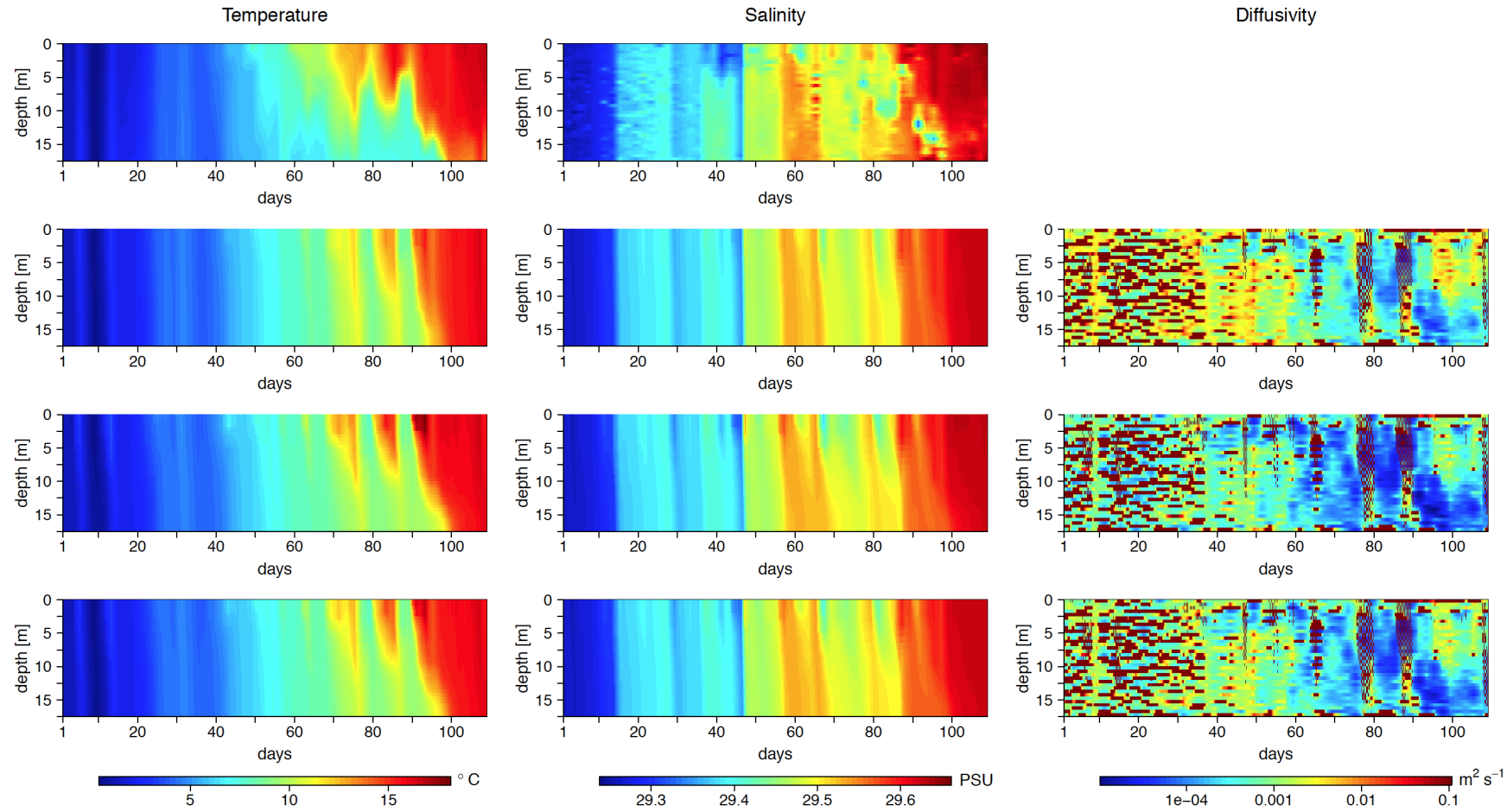

Figure S 14. Temporal evolution of temperature, salinity, and diffusivity profiles in mesocosm \#5 of KOSMOS 2013. The first row shows observed temperature and salinity; the following rows show simulated temperature, salinity, and diffusivity for a) the salinity optimisation (second row), b) the temperature optimisation (third row), and c) the optimisation that depends on both temperature and salinity (fourth row). 

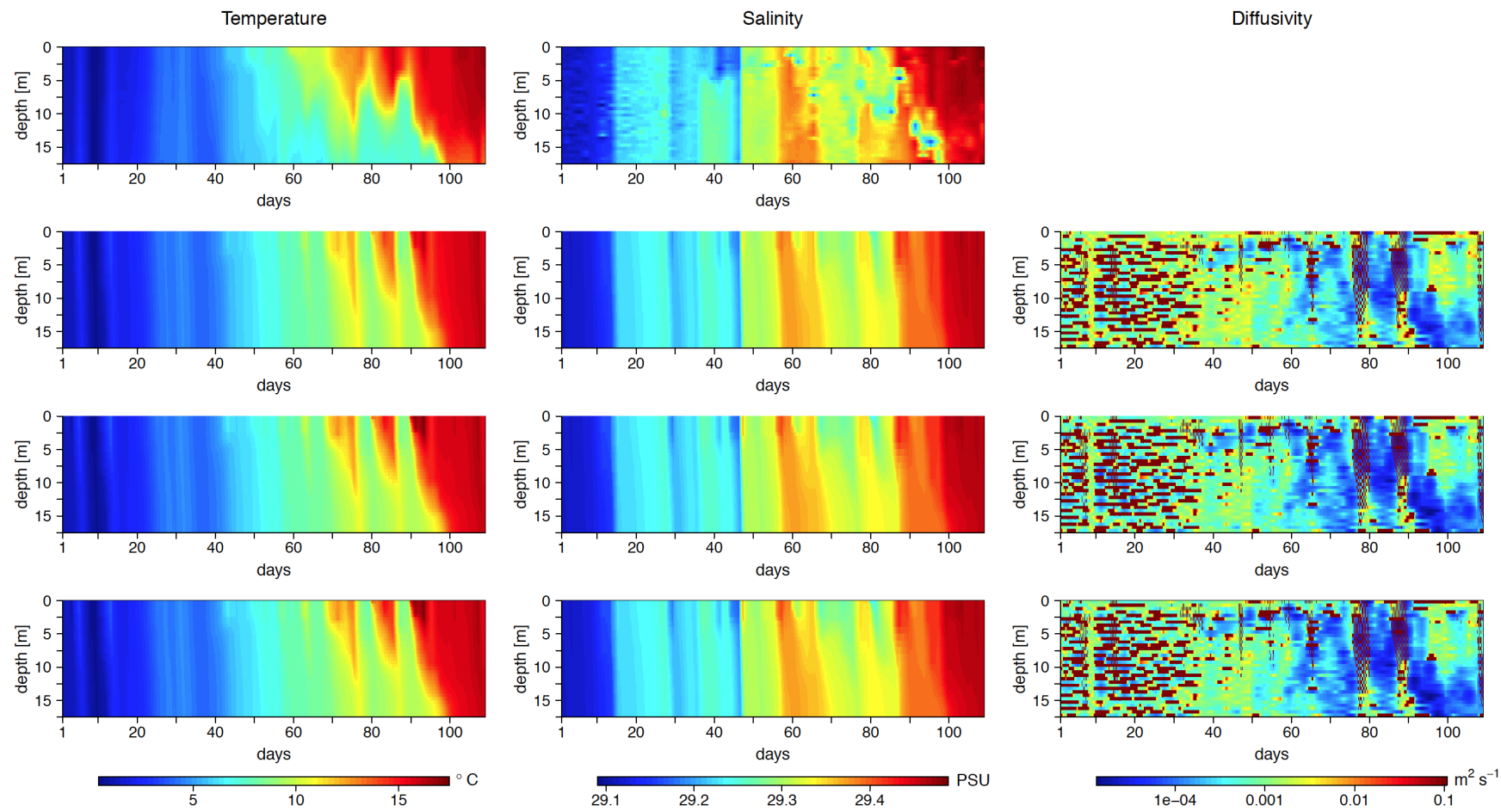

Figure S 15. Temporal evolution of temperature, salinity, and diffusivity profiles in mesocosm \#6 of KOSMOS 2013. The first row shows observed temperature and salinity; the following rows show simulated temperature, salinity, and diffusivity for a) the salinity optimisation (second row), b) the temperature optimisation (third row), and c) the optimisation that depends on both temperature and salinity (fourth row).
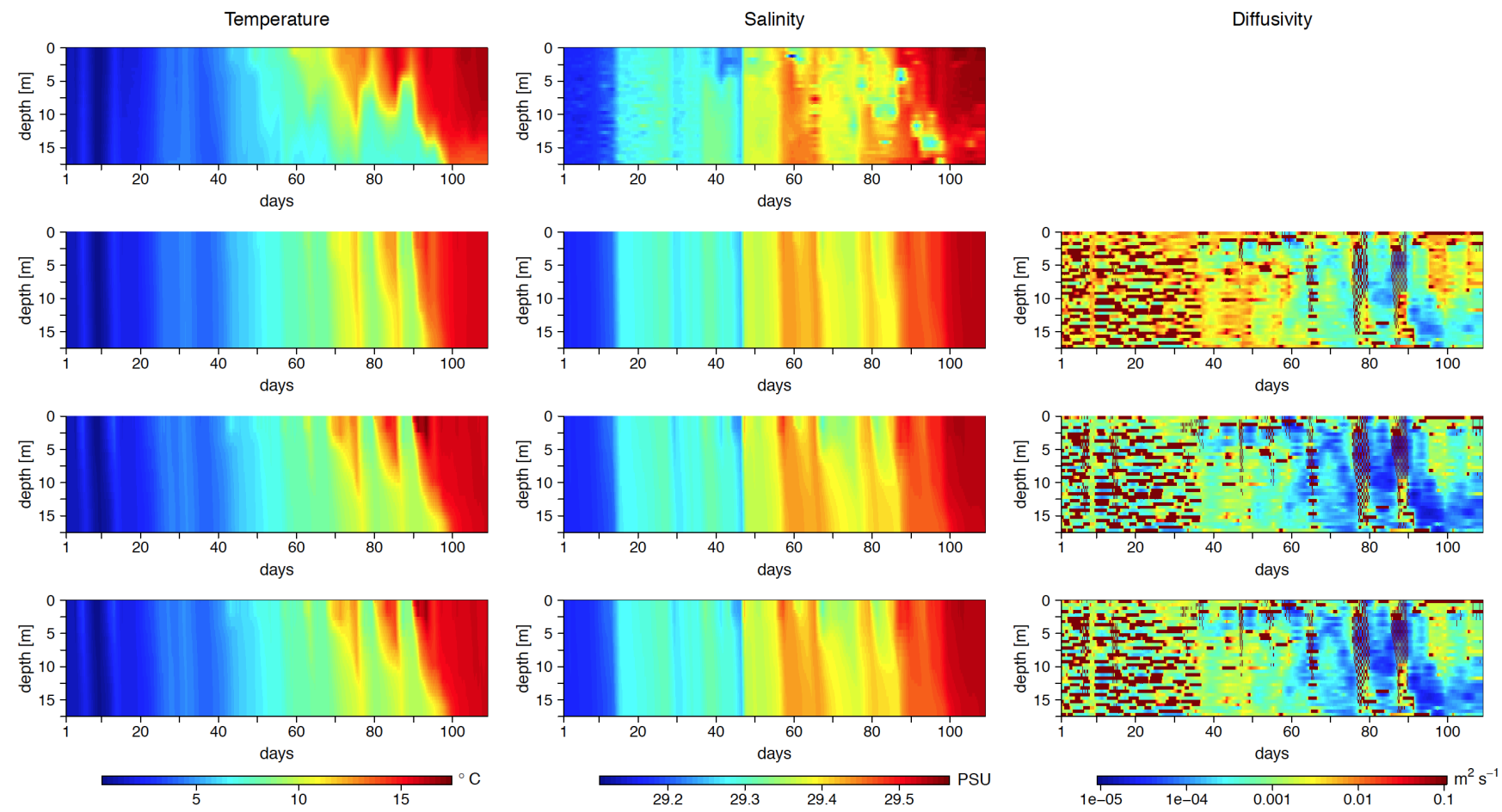

Figure S 16. Temporal evolution of temperature, salinity, and diffusivity profiles in mesocosm \#7 of KOSMOS 2013. The first row shows observed temperature and salinity; the following rows show simulated temperature, salinity, and diffusivity for a) the salinity optimisation (second row), b) the temperature optimisation (third row), and c) the optimisation that depends on both temperature and salinity (fourth row). 

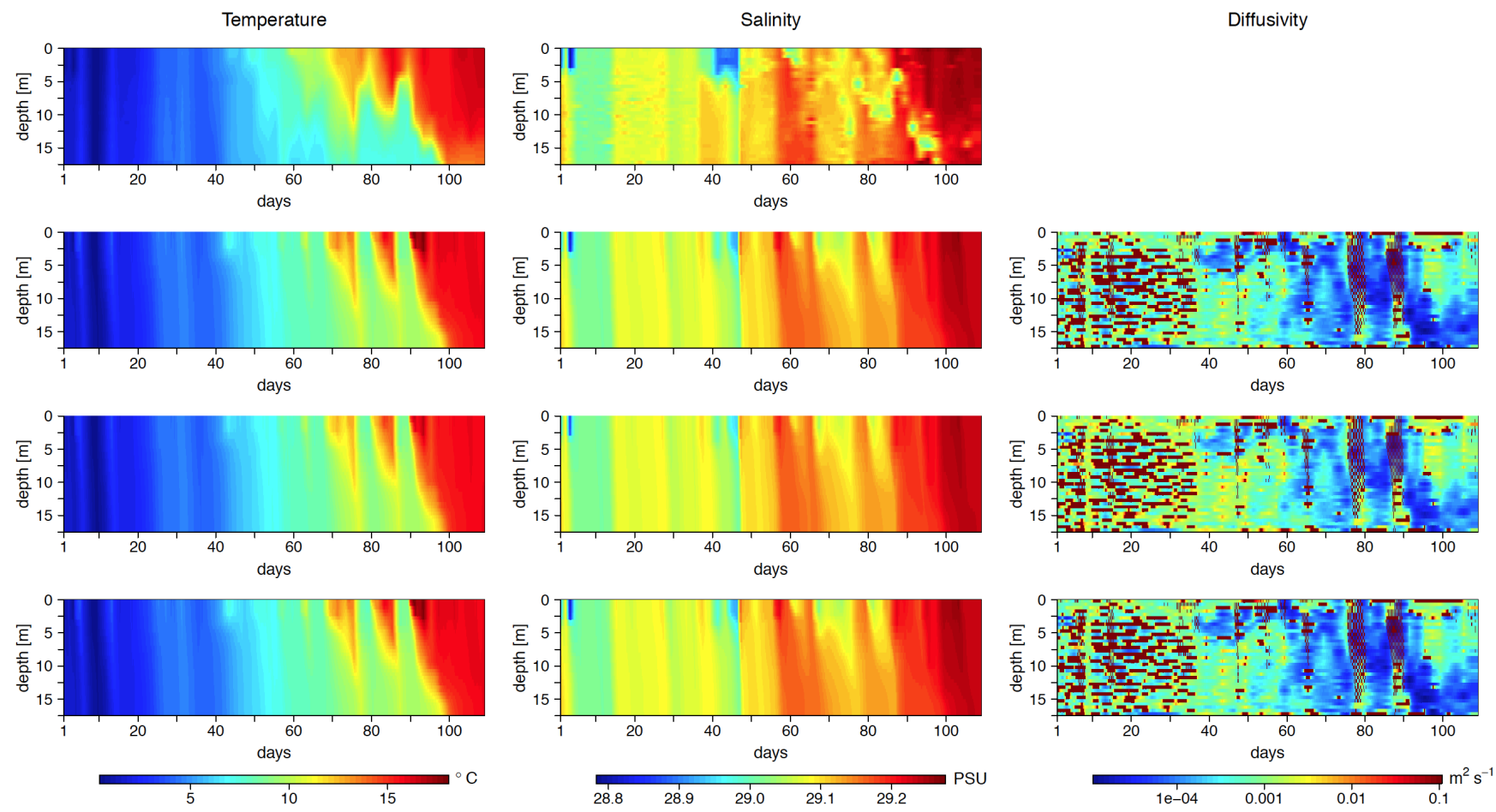

Figure S 17. Temporal evolution of temperature, salinity, and diffusivity profiles in mesocosm \#8 of KOSMOS 2013. The first row shows observed temperature and salinity; the following rows show simulated temperature, salinity, and diffusivity for a) the salinity optimisation (second row), b) the temperature optimisation (third row), and c) the optimisation that depends on both temperature and salinity (fourth row).
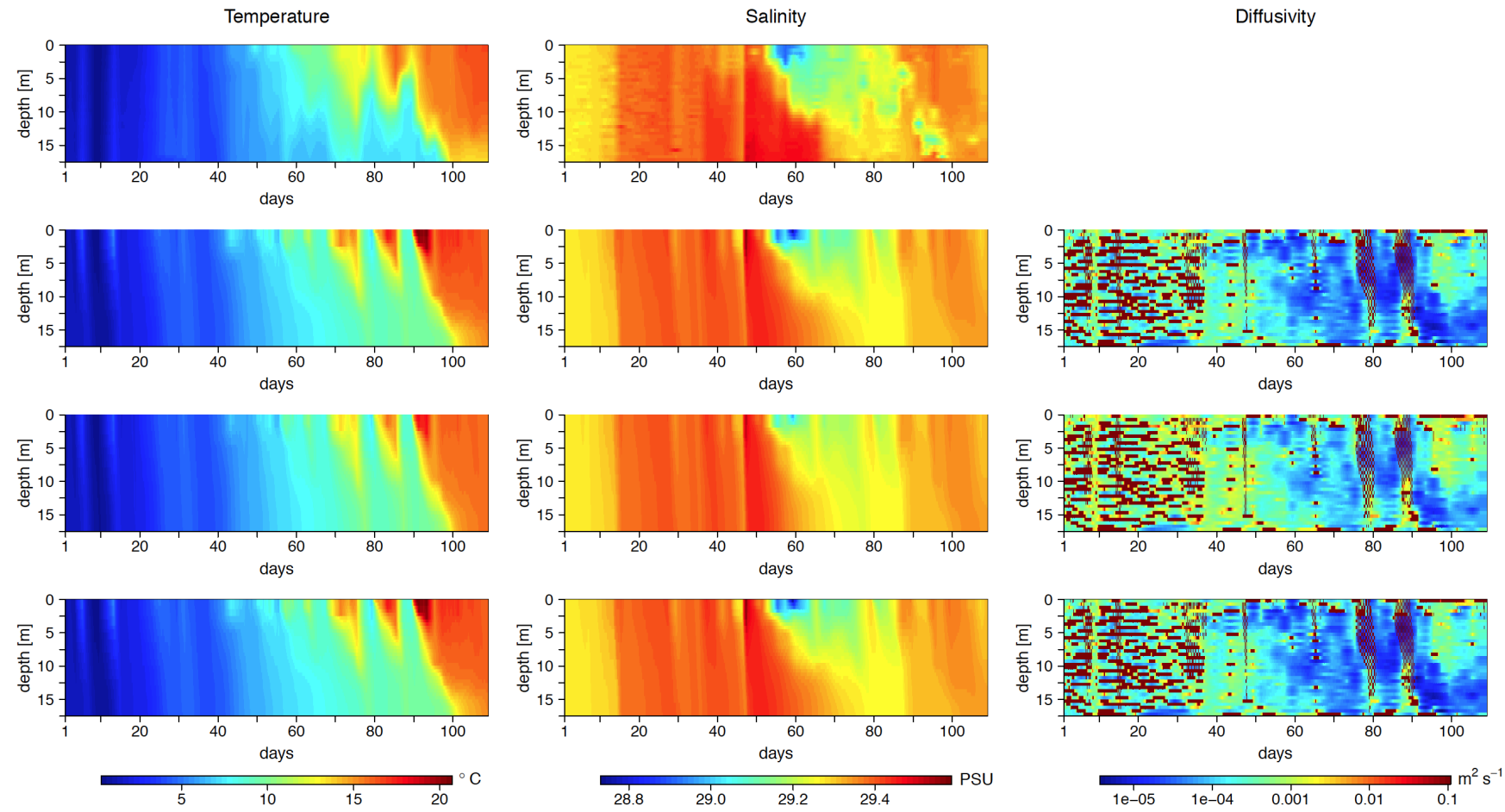

Figure S 18. Temporal evolution of temperature, salinity, and diffusivity profiles in mesocosm \#9 of KOSMOS 2013. The first row shows observed temperature and salinity; the following rows show simulated temperature, salinity, and diffusivity for a) the salinity optimisation (second row), b) the temperature optimisation (third row), and c) the optimisation that depends on both temperature and salinity (fourth row). 

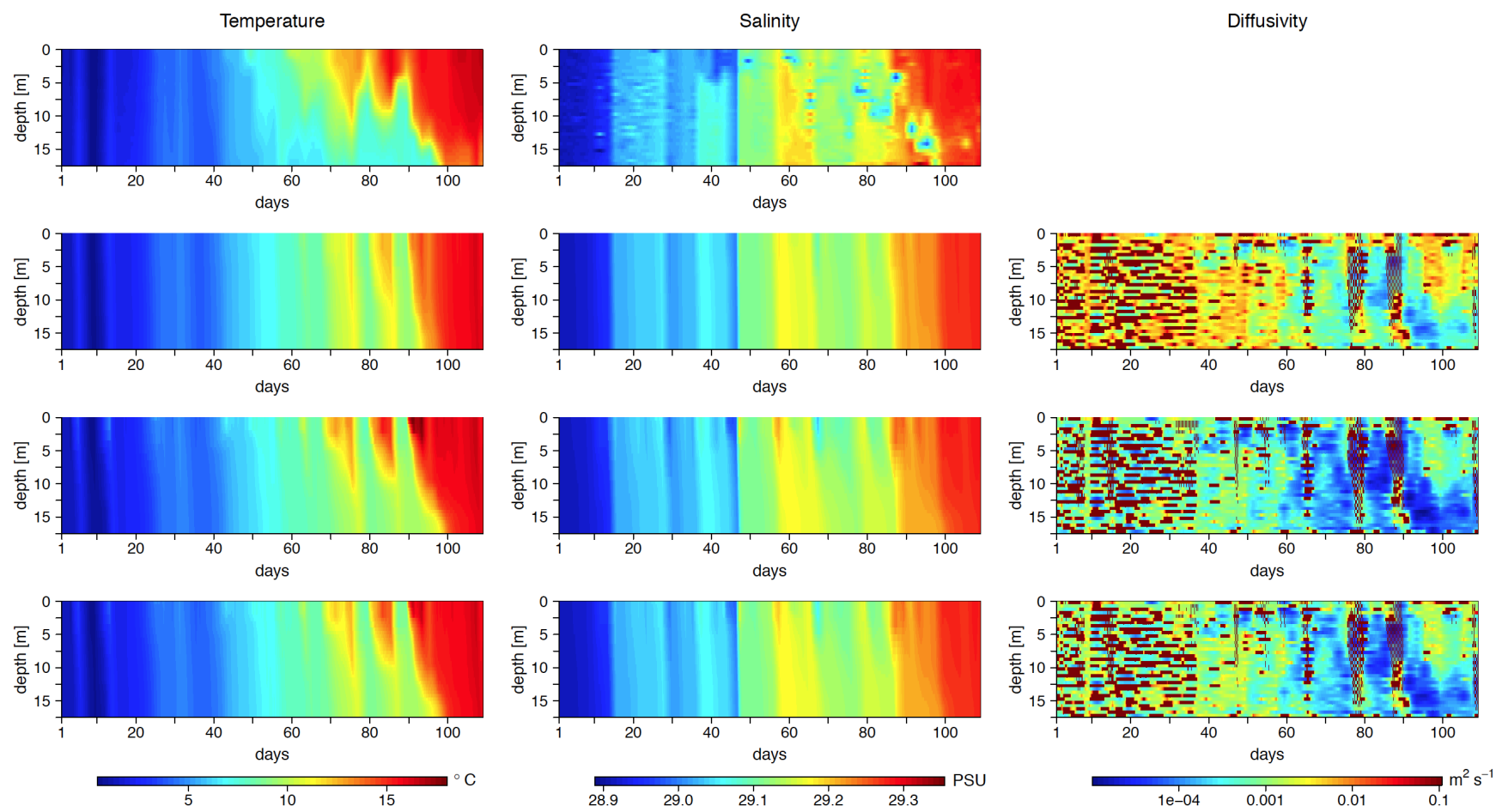

Figure S 19. Temporal evolution of temperature, salinity, and diffusivity profiles in mesocosm \#10 of KOSMOS 2013. The first row shows observed temperature and salinity; the following rows show simulated temperature, salinity, and diffusivity for a) the salinity optimisation (second row), b) the temperature optimisation (third row), and c) the optimisation that depends on both temperature and salinity (fourth row). 